\title{
De artrópodos y plantas: Diversidad de la artropodofauna en un gradiente de vegetación en Los Llanos riojanos, Argentina
}

\author{
Florencia Baudino ${ }^{1, \bigotimes}$; Nicolas R. Cecchetto ${ }^{2}$; Liliana M. Buffa ${ }^{3}$ \& Andrés M. \\ VISINTIN ${ }^{3 / 4}$ \\ ${ }^{1}$ Laboratorio Ecotono. Universidad Nacional del Comahue (INIBIOMA-CONICET). ${ }^{2}$ Universidad Nacional del Comahue \\ (INIBIOMA-CONICET). ${ }^{3}$ Cátedra de Entomología. Centro de Investigaciones Entomológicas (CIEC). Facultad de Ciencias \\ Exactas Físicas y Naturales (FCEFyN). Universidad Nacional de Córdoba (UNC). ${ }^{4}$ Instituto de Biología de la Conservación y \\ Paleobiología (IBiCoPa), Centro de Investigación e Innovación Tecnológica (CENIIT), Universidad Nacional de La Rioja.
}

\begin{abstract}
Resumen. Dado el rol fundamental de los artrópodos y los insectos en el funcionamiento de los ecosistemas áridos, para manejar estos ambientes resulta necesario conocer su relación con las características de la vegetación local. Dentro de la región fitogeográfica del Chaco, en Los Llanos de la provincia de La Rioja, Argentina, existen grandes cambios en la vegetación en distancias cortas, lo que determina un gradiente de heterogeneidad vegetal. El objetivo de este estudio fue analizar la respuesta de los artrópodos epígeos a estos cambios. En este gradiente se seleccionaron 3 ambientes (Salinas, Interfaz y Monte) en los que se recolectaron artrópodos y, dentro de éstos, insectos mediante trampas de caída durante 7 días en la temporada cálida. Se determinó la abundancia total de artrópodos e insectos y la riqueza de especies/morfoespecies de insectos. Se evaluó la asociación entre los ambientes y la abundancia y riqueza de especies mediante un ANOVA de medidas repetidas. Para evaluar la completitud del muestreo se utilizaron estimadores de riqueza no-paramétricos (Chao ${ }_{1}$ Jackniffe $_{1}$ y Bootstrap) y curvas de acumulación de especies. Para evaluar la estructura de la comunidad se usaron curvas de rango-abundancia y análisis multivariados. Se recolectaron en total 8380 artrópodos, de los cuales 5902 fueron insectos que se agruparon en 14 órdenes, 31 superfamilias, 35 familias y 114 especies/morfoespecies. El ambiente con mayor heterogeneidad vegetal (Monte) presentó la mayor abundancia de artrópodos y la mayor abundancia y riqueza de insectos. Las familias de insectos más abundantes y de mayor riqueza fueron Formicidae (Hymenoptera) y Tenebrionidae (Coleoptera). Chao ${ }_{1}$ y Bootstrap fueron los mejores estimadores de riqueza. En este estudio se encontró que la abundancia, la riqueza y la composición de especies de artrópodos, y particularmente insectos, estuvo asociada a un gradiente de vegetación.
\end{abstract}

[Palabras clave: variabilidad, entomofauna epígea, indicadores, suelo, región chaqueña, La Rioja]

\begin{abstract}
AвstRACT. Of arthropods and plants: Arthropod fauna diversity in a vegetation gradient in Los Llanos of La Rioja province (Argentina). Given the fundamental role of arthropods and insects in the functioning of arid ecosystems, it is of key importance to understand their relationships with the environment, particularly for the development of future conservation efforts. Within the phytogeographic region of Chaco, in Los Llanos (La Rioja province, Argentina), great changes in vegetation occur within short distances, determining a pronounced gradient of plant heterogeneity. The aim of this study was to analyse the response of epigaeic arthropods to these changes. In this gradient, three environments were selected (Salinas, Interface and Monte), in which we collected arthropods and insects using fall traps during seven days during the warm season. Total abundance of arthropods and insects, and richness of insect species/morpho-species were determined. The association between the environments and the abundance and richness of species was evaluated by repeated measurements ANOVA. Nonparametric richness estimators ( $\mathrm{Chao}_{1}$, Jackniffe1 and Bootstrap) and their species accumulation curves were used to test sampling completeness. To assess the structure of the insect community we used range-abundance curves and multivariate analysis. A total 8380 arthropods was collected, of which 5902 were insects grouped into 14 orders, 31 superfamilies, 35 families and 114 species/morphospecies. The environment with the greatest plant heterogeneity (Monte) presented the greatest abundance of arthropods and the greatest abundance and richness of insects. The most abundant and richest insect families were Formicidae (Hymenoptera) and Tenebrionidae (Coleoptera). Chao ${ }_{1}$ and Bootstrap were the best richness estimators. In this study, we found that the abundance, richness and composition of arthropod species, particularly insects, were associated to a vegetation gradient.
\end{abstract}

[Keywords: variability, epigaeic entomofauna, indicators, soil, Chaco ecoregion, La Rioja]

Editor asociado: Ricardo Grau
Recibido: 7 de Enero de 2019

Aceptado: 10 de Noviembre de 2019 


\section{INTRODUCCIÓN}

La heterogeneidad ambiental que genera la vegetación aumenta la diversidad del ecosistema (Tews et al. 2004; Stein et al. 2014). Ambientes con escasa cobertura vegetal exponen al suelo a la erosión, lo que impide que se establezcan nuevas especies vegetales $y$, en consecuencia, otros taxa (Zuazo and Pleguezuelo 2009). En cambio, los ambientes con mayor heterogeneidad y una estructuración vertical y horizontal de la vegetación más compleja promueven mayores oportunidades de refugio, alimento y reproducción para los organismos, por lo cual suelen presentar un incremento en el número de especies (Tews et al. 2004; Stein et al. 2014). En particular, en ambientes áridos y semiáridos, la presencia de estructuras clave como las especies leñosas genera gradientes ambientales; esto aumenta la heterogeneidad espacial, lo que modifica los patrones de distribución de otras especies (Rossi and Villagra 2003; Álvarez et al. 2009). Si bien existe una relación positiva entre la heterogeneidad ambiental producida por la vegetación y la diversidad de animales, difiere según los grupos taxonómicos, ya que la mayoría de los estudios están sesgados hacia grupos de vertebrados o hábitats con influencia antropogénica (Tews et al. 2004).

Los artrópodos epígeos son uno de los componentes principales en los ecosistemas $y$, especialmente, en el suelo. Su gran diversidad en el suelo está correlacionada con la heterogeneidad del hábitat, la cual favorece una mayor disponibilidad de recursos e interacciones interespecíficas (Bardgett and Wardle 2010). En ambientes áridos y semiáridos, los artrópodos epígeos tienen roles clave como descomponedores, herbívoros, granívoros y depredadores, controlando el ciclo de nutrientes y los flujos de energía en diferentes niveles de la cadena trófica (Ayal 2007; Araujo et al. 2012). Estos ambientes se caracterizan por una dominancia de Hymenoptera (Formicidae en particular), Colembolla y Acari, y en menor abundancia Aranae, Solifugae, Scorpionidae, Coleoptera y Diptera (Lagos 2004; Flores et al. 2004; Peñaloza et al. 2012; González Reyes et al. 2012). Los ácaros y colémbolos son importantes descomponedores de hojarasca y favorecen la dinámica y el funcionamiento del suelo (Gergócs and Hufnagel 2016), mientras que las arañas y escorpiones se caracterizan por sus hábitos depredadores, con lo que modifican la dinámica y la estructura de los ensambles por sus relaciones con otros taxa (Tripplehorn and Johnson 2005). Dentro de los insectos, los Scarabaeidae y Staphylinidae promueven el ciclado de nutrientes en el suelo a través de sus hábitos descomponedores (Tripplehorn and Johnson 2005), mientras que otros grupos como Formicidae, al ser tan diversos, poseen una variabilidad amplia de gremios con distintos requerimientos ecológicos, que influyen a diversas escalas dentro del ecosistema (Hölldobler and Wilson 1990). Todos estos grupos, por sus importantes funciones ecosistémicas y por su sensibilidad a cambios ambientales, se destacan como grupos indicadores, ya sea del tipo de hábitat, de la calidad del ecosistema o de los cambios ambientales, por lo que fueron incluidos en planes de manejo y monitoreo (Gerlach et al. 2013).

A pesar de la importancia de los artrópodos e insectos epígeos en regular la dinámica del suelo, registrar el total de especies en estos grupos hiperdiversos (Martín-Piera and Lobo 2000) es muy difícil (Moreno 2001; Magurran 2004; Gotelli and Colwell 2011). Los estimadores no paramétricos de riqueza son útiles para cuantificar la biodiversidad en estas comunidades hiperdiversas (Moreno 2001; Magurran 2004). El análisis de los estimadores de riqueza nos permite evaluar la eficiencia o completitud del muestreo, estimar la riqueza total y además comparar la riqueza de especies entre sitios que posean el mismo esfuerzo de muestreo (Magurran 2004; Chao et al. 2009). Aunque no existe un estimador idóneo para todas las situaciones o específico para taxa particulares (Walther and Moore 2005), diversos estudios propusieron estimadores para sitios particulares (Brose [2002], para ambientes acuáticos, Chao; Hortal et al. [2006], para bosques, estimadores como ACE, $\mathrm{Chao}_{1}$, Jackknife ${ }_{1} \mathrm{y}_{2}$, y Bootstrap; Basualdo [2011], para ríos, estimadores como Jackknife, Chao y ACE). Considerando la escasez de conocimiento en los patrones de diversidad y composición del ensamble de artrópodos e insectos en particular en Los Llanos riojanos, analizar los estimadores de riqueza, nos brindan no sólo la posibilidad de proponer un buen estimador para esta zona árida, sino de estimar la riqueza total y generar muestreos completos. Estas ventajas permitirían hacer más eficientes los futuros estudios en la zona que son de vital importancia para establecer planes de manejo y conservación.

La variación de la diversidad de especies a lo largo de gradientes de vegetación en 
zonas áridas de la Argentina está muy estudiada, en particular en las unidades biogeográficas del Monte (Roig-Juñent et al. 2001; Flores et al. 2004; Roig et al. 2009) y del Chaco árido (Gardner et al. 1995; Lagos 2004). Sin embargo, en pocos casos se analizó la variación de la diversidad de artrópodos en la zona de transición entre las unidades de Chaco y Monte. Estudios biogeográficos encontraron una zona de confluencia para dos especies de escorpiones en la región de Los Llanos (Acosta 1995; Mattoni and Acosta 1997), mientras que otro estudio describe una mayor riqueza de insectos para la provincia biogeográfica del Chaco respecto a la de Monte, aunque esta última presenta un mayor número de endemismos (Roig-Juñet et al. 2001). En particular, en la provincia de La Rioja, en el Parque Nacional Talampaya (Monte) se encontraron diferencias en la diversidad y la composición del ensamble de artrópodos e insectos en ambientes con distinta composición florística (Peñaloza et al. 2012). Aunque el Chaco árido presenta diversos endemismos y faunas con historias biogeográficas complejas, en la actualidad enfrenta una gran pérdida de biodiversidad por causas antrópicas (Biani et al. 2004; Grau et al. 2011), lo cual podría generar cambios estructurales y funcionales irreversibles en el ecosistema. Conocer la diversidad de especies en estas áreas y su relación con las formaciones vegetales locales es fundamental para establecer zonas prioritarias de conservación, además de generar herramientas para planes de manejo sustentable para los pobladores de la zona (Natenzon 1994; Coirini and Karlin 2000; Coirini et al. 2010).

El objetivo general de este trabajo fue analizar la variación del ensamble de artrópodos con énfasis en los insectos, en un gradiente de vegetación de una zona árida como Los Llanos de La Rioja. Particularmente, nos preguntamos: a) ¿Cuál es el mejor estimador de riqueza de insectos en una zona árida como Los Llanos riojanos?, b)¿Existen diferencias en la riqueza de insectos entre los ambientes y estaciones?, c) ¿Existen diferencias en la abundancia de artrópodos y particularmente de insectos entre los ambientes? d) ¿Cómo es la relación de la riqueza y abundancia de insectos entre los ambientes?, e) Considerando la composición del ensamble de insectos: ¿Cómo es la dominancia entre los distintos niveles taxonómicos (órdenes, familias, especies)?, ¿Qué grado de similitud tiene la composición de familias entre los ambientes y estaciones?
Considerando que la heterogeneidad vegetal promueve una mayor diversidad de especies, se esperaría que, en un gradiente de vegetación en una zona árida, la diversidad (abundancia y riqueza) de artrópodos, y en particular de insectos, aumente con la complejidad vegetal (e.g., presencia de especies leñosas, diversos estratos vegetales, diversidad de especies vegetales). Por otro lado, considerando las características particulares de cada ambiente que generan distintos recursos para los insectos y los requerimientos específicos de las especies, esperamos encontrar ensambles con distinta composición taxonómica en los distintos ambientes.

\section{Materiales y Métodos}

\section{Área de estudio}

La región de Los Llanos, en la provincia de La Rioja, pertenece a la zona más árida de la región fitogeográfica del Chaco árido, particularmente a la región chaqueña occidental (Ragonese and Castiglioni 1970; Morello et al. 1973). Posee un clima subtropical seco o semidesértico, con precipitaciones medias que varían entre 300 y 500 mm/año de oeste a este, y temperaturas medias mensuales de $26{ }^{\circ} \mathrm{C}$ en el mes más cálido y de $12{ }^{\circ} \mathrm{C}$ en el mes más frío, con importantes oscilaciones térmicas diarias (Morello et al. 1973; Karlin et al. 2013). El trabajo de campo se realizó en el Departamento General Belgrano (30³7'50' S - 66 $\left.16^{\prime} 3^{\prime \prime} \mathrm{O}\right)$, al sudeste de la provincia de La Rioja (Figura 1). Se seleccionaron tres áreas sobre la base de la estructura y diversidad de la vegetación dominante, resultando en tres ambientes denominados: 1) Salinas, 2) Interfaz Salinas-Monte y 3) Monte. El ambiente de Salinas (Figura 1b) presenta una alta temperatura media y un suelo arcilloso pobre en materia orgánica, con escaso desarrollo de vegetación (Ruiz Posse et al. 2007; Coirini et al. 2010). La comunidad vegetal dominante posee especies halófitas de poca altitud como jumeales de Allenrolfea patagonica (Moq.) Kuntze, Heterostachys ritteriana (Moq.) Ung.Sternb., Atriplex argentina Speg., Salicornia ambigua Michx, pasto raíz (Trichloris crinita (Lag.) Parodi) y por algunos cardonales de Stetsonia coryne (Salm-Dyck) Britton and Rose (Ragonese and Castiglioni 1970; Coirini et al. 2010; Biurrun et al. 2012) y está incluido dentro de las Salinas Grandes (Morello et al. 1973; Oyarzabal et al. 2018). Por otro lado, el ambiente Interfaz (Figura 1c), ubicado entre los ambientes Salinas y Monte, dominado por 
a)

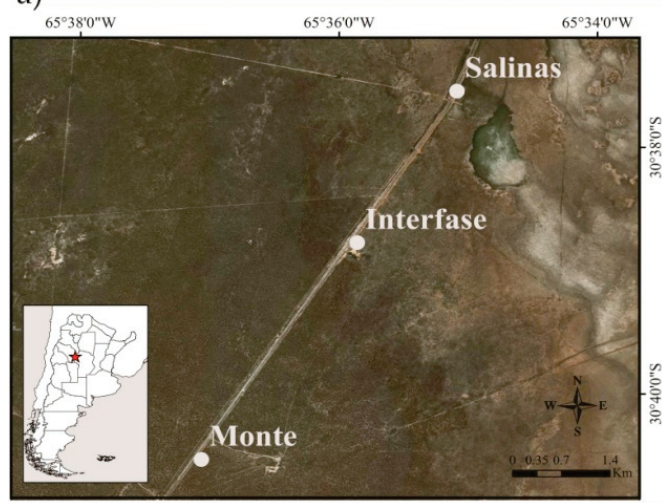

c)

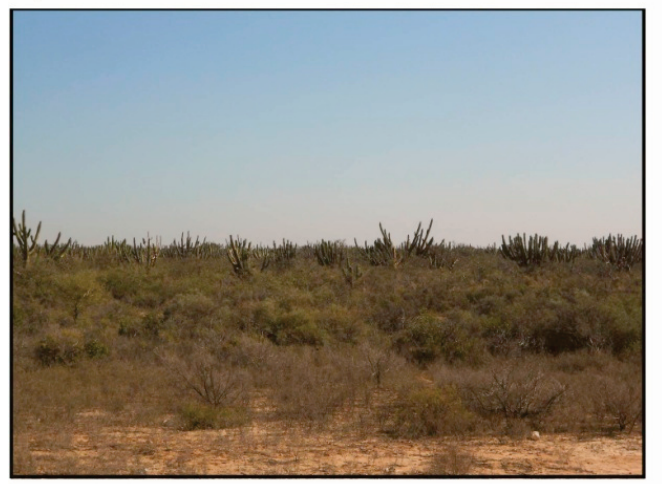

b)

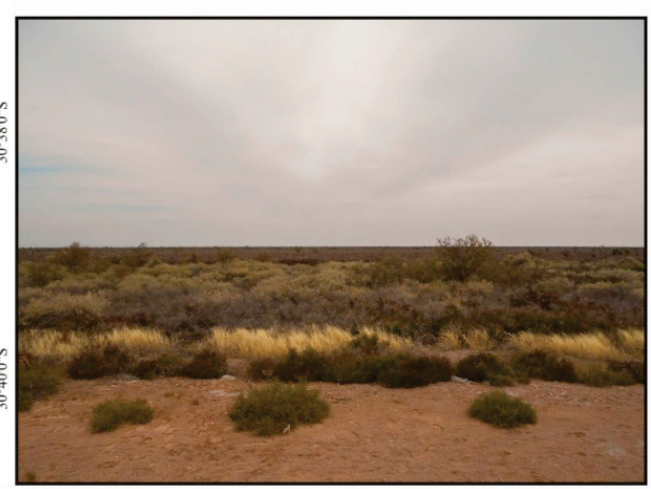

d)

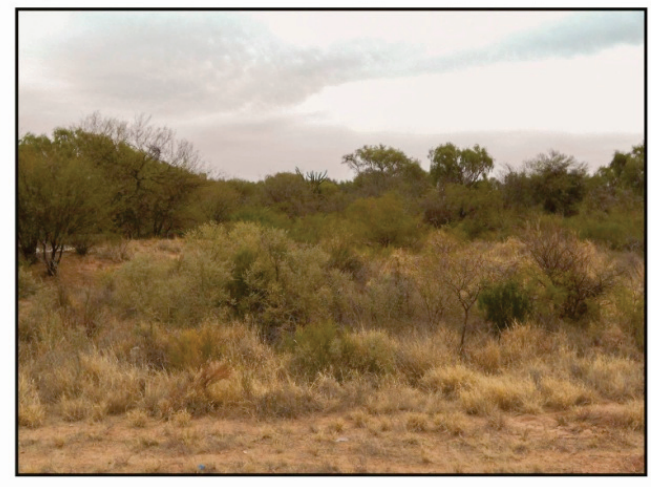

Figura 1. a) Localización del área de estudio en la región de Los Llanos (La Rioja), indicando los tres ambientes de muestreo, b) Ambiente Salinas (30.62573 S - 65.58479 O), c) Ambiente Interfaz (30.64622 S - 65.59765 O), d) Ambiente Monte (30.67553 S - 65.61777 O).

Figure 1. a) Location of the study area in Los Llanos region (La Rioja province) showing the three sampling sites, b) Salinas environment (30.62573 S - 65.58479 W), c) Interface environment (30.64622 S - $65.59765 \mathrm{~W})$, d) Monte environment (30.67553 S - $65.61777 \mathrm{~W})$.

un abundante estrato arbustivo de cardones y jarillas (Larrea divaricata Cav.) y un estrato más bajo de plumerillo (Trichloris crinita (Lag.) Parodi) y avenilla (Gouinia paraguayensis (Kuntze) Parodi var.), presenta una mayor estructuración vertical que el ambiente Salinas, pero menor que el Monte, ya que no tiene especies leñosas de gran envergadura. Sin embargo, comparte especies vegetales con los otros ambientes: jumeales con Salinas y jarillas con el Monte. Por último, el ambiente Monte (Figura 1d) presenta mayor cantidad de materia orgánica, menor temperatura media y la mayor complejidad en la estructura vegetal de los tres ambientes, con una cobertura foliar densa. Predominan las especies leñosas como algarrobos (Prosopis flexuosa), quebrachos (Aspidosperma quebracho-blanco Schltdl), tintitaco (Prosopis torquata (Cav. Ex Lag.) DC.) y chañar (Geoffrea decorticans (Gillies ex Hook. and Arn.) Burkart), generando un estrato arbóreo que oscila entre 3 y $6 \mathrm{~m}$ y un estrato arbustivo entre 0.5 y $3 \mathrm{~m}$ de altura de lata (Mimozyganthus carinatus (Griseb.) Burkart), brea (Cercidium praecox (Ruiz and Pav. Ex Hook.) Harms) y jarilla. El estrato más bajo está formado por escasos plumerillo y avenilla, sin presencia de especies halófitas (Morello et al. 1973; Biurrun et al. 2012; Karlin et al. 2013; Oyarzabal et al. 2018).

\section{Recolección de artrópodos}

Se recolectaron artrópodos epígeos mediante trampas de caída o pitfall de plástico (diámetro, $8 \mathrm{~cm}$; profundidad, $11 \mathrm{~cm}$ ). Se utilizaron 4 cuatro trampas por transecta dispuestas cada $5 \mathrm{~m}$ entre sí, y se colocaron cuatro transectas en cada ambiente (Salinas, Interfaz y Monte), dispuestas paralelamente cada $50 \mathrm{~m}$ entre sí y a 200 m de la Ruta Nacional N ${ }^{\circ} 77$ (total de trampas $=48$ ). Las trampas se llenaron con 200 $\mathrm{mL}$ de etilenglicol diluido (20\%) y una gota de detergente para reducir la tensión superficial. El etilenglicol impide la evaporación y permite conservar los organismos capturados. Las trampas permanecieron activas en el campo durante 7 días en primavera 
y verano (noviembre y marzo de 2013, respectivamente), ya que en la temporada cálida se encuentra la mayor abundancia de artrópodos epígeos (Flores et al. 2004). El contenido de las trampas fue preservado en alcohol $80 \%$. Los artrópodos fueron separados en órdenes y los insectos se clasificaron hasta el nivel taxonómico más detallado posible mediante claves especializadas (Tripplehorn and Johnson 2005; Fernández and Sharkey 2006; Fisher and Cover 2007). Se acondicionó y depositó el material en las colecciones entomológicas de las cátedras de EntomologíaCentro de Investigaciones Entomológicas de Córdoba, Universidad Nacional de Córdoba (UNC) y de la Universidad Nacional de La Rioja (UNLaR).

\section{Análisis de datos}

Para estimar la riqueza de especies se utilizaron tres estimadores no paramétricos: Chao $_{1}$ Jackknife $_{1}$ y Bootstrap (Moreno 2001; Magurran 2004). Se consideró la riqueza de especies como el número total de especies/ morfoespecies de los órdenes más abundantes de insectos en cada ambiente y estación de muestreo. Se evaluó la eficiencia de cada estimador utilizando los valores de sesgo y precisión (Walther and Moore 2005) mediante la relación entre la riqueza estimada (Sest) y la riqueza observada (Sobs) (Brose 2002). Para evaluar el esfuerzo de muestreo y estimar la riqueza total (Stot) se utilizaron datos de presencia/ausenciadeespecies/morfoespecies y se construyeron curvas de acumulación de especies utilizando el módulo de regresión no lineal en el programa Infostat (Di Rienzo et al. 2009). Se consideró como mejor estimador al que tuviera una curva de acumulación con una alta tasa de crecimiento inicial y una asíntota definida (Soberon and Llorente 1993; Chao et al. 2009; Gotelli and Colwell 2011). El esfuerzo de muestreo fue el número de transectas en cada ambiente, el cual fue aleatorizado 50 veces para evitar que el orden de agregación de las muestras afectara la forma de las curvas de acumulación de especies (Gotelli and Colwell 2001). Para ello se utilizó el programa Species Diversity and Richness (Henderson and Seaby 1997). A fin de evaluar la relación entre la abundancia y la riqueza de insectos entre los ambientes se realizaron curvas de rango-abundancia y se ajustaron los siguientes modelos teóricos: serie geométrica, serie logarítmica, log-normal y vara quebrada (Moreno 2001; Magurran 2004; Maurer and McGill 2011) utilizando el programa Species
Diversity and Richness (Henderson and Seaby 1997). Se asignaron categorías de abundancia (en escala logarítmica) a las especies y las frecuencias observadas se compararon con las esperadas bajo el modelo al que se pretendió ajustar mediante una prueba de $\chi^{2}$. Finalmente, se probó normalidad y heterocedasticidad de los residuos mediante las pruebas de ShapiroWilks y Levene, respectivamente, utilizando el programa Infostat (Di Rienzo et al. 2009).

Para evaluar diferencias en la abundancia de artrópodos e insectos y en la riqueza de especies de insectos entre ambientes y estaciones se realizaron análisis de varianza (ANOVA) de medidas repetidas. Se consideró la abundancia como el número total de individuos recolectados en cada ambiente y estación de muestreo. En caso de encontrarse diferencias significativas, se realizaron pruebas de Tukey a posteriori de comparaciones múltiples utilizando el programa SPSS 17.0 (Released 2008).

Para evaluar la dominancia de los distintos grupos taxonómicos de insectos se determinaron las siguientes categorías de dominancia (CD): eudominantes $(C D>10 \%)$, dominantes $(5 \%<\mathrm{CD} \leq 10 \%)$, subdominantes $(2 \%<\mathrm{CD} \leq 5 \%)$, eventuales $(1 \%<\mathrm{CD} \leq 2 \%)$ o raras (CD $\leq 1 \%$ ) (Cardoso et al. 2011). Se consideraron las abundancias relativas respecto a la total para cada grupo taxonómico. Para evaluar las diferencias en la composición del ensamble de insectos entre ambientes y estaciones de muestreo se realizó un análisis de conglomerados (cluster) que examina distancia y agrupamiento en base a datos de abundancia mediante el índice de Bray-Curtis. Para evaluar la asociación de las familias de insectosconambientes y estaciones particulares realizamos un análisis de correspondencias (AC) basado en datos de abundancia. En ambos análisis se utilizó la abundancia de las familias más representativas de Hymenoptera, Coleoptera, Díptera y Hemiptera, previamente transformados a logaritmo, mediante el programa MVSP (Kovach 1999). Se analizó la diversidad $\left(\mathrm{H}^{\prime}\right)$ y la equidad ( $\left.\mathrm{H}^{\prime} \mathrm{eq}\right)$ de insectos entre los ambientes y estaciones mediante el índice de Shannon-Wienner y la dominancia a través del índice de Simpson (DS) (Moreno 2001; Maurer and McGill 2011) con el programa MSVP (Kovach 1999). Los valores obtenidos para cada uno de los ambientes fueron comparados mediante Kruskal-Wallis utilizando el programa Infostat (Di Rienzo et al. 2009). 


\section{RESUltados}

Se registró un total de 8380 artrópodos en los tres ambientes y en las dos estaciones. Dos de los tres estimadores de riqueza utilizados fueron más apropiados para analizar la diversidad de insectos en Los Llanos riojanos durante la temporada cálida: $\mathrm{Chao}_{1}$ obtuvo los menores valores de sesgo y la mejor estimación de la riqueza observada, mientras que Bootstrap tuvo la mayor precisión. Jackknife ${ }_{1}$ tuvo valores intermedios de sesgo y precisión entre los otros estimadores (Tabla 1). Por otro lado, las curvas de acumulación de especies, basadas en los estimadores de riqueza, no presentaron una asíntota definida en ninguno de los tres ambientes (Figura 2). Las curvas de rango-abundancia de las especies/morfoespecies de insectos para cada ambiente durante la temporada cálida presentaron un gran número de especies con abundancia intermedia y pocas especies de escasa (raras) o gran abundancia (Figura 3). El mejor ajuste se logró con el modelo lognormal truncado para los tres ambientes: Salinas $\left(\chi^{2}=4.039 ; P=0.854\right)$, Interfaz $\left(\chi^{2}=6.446\right.$; $P=0.695)$ y Monte $\left(\chi^{2}=2.719 ; P=0.974\right)$, mientras que este último ajustó, además, al modelo log series $\left(\chi^{2}=7.880 ; P=0.546\right)$.

Tabla 1. Evaluación de los estimadores de riqueza no paramétricos $\mathrm{Chao}_{1}$, Jackknife ${ }_{1}$ y Bootstrap mediante la comparación del sesgo y de la precisión de las especies registradas en los tres ambientes (Salinas, Interfaz, Monte) en la región de Los Llanos (La Rioja) durante la temporada cálida (primavera y verano). Sobs=Riqueza observada, Stot=Riqueza total.

Table 1. Assessment of the non-parametric species richness estimators $\mathrm{ChaO}_{1}$, Jackknife ${ }_{1}$ y Bootstrap comparing bias and accuracy of the registered species in the three environments (Salinas, Interface, Monte) in Los Llanos region (La Rioja province) during the warm season (Spring and Summer). Sobs=Observed species richness, Stot=Total species richness.

\begin{tabular}{llccc}
\hline \multirow{4}{*}{ Muestreo } & \multicolumn{3}{c}{ Salinas } & \multicolumn{2}{c}{ Interfaz Monte } \\
\hline \multirow{5}{*}{ Estimación } & Sobs & 56 & 70 & 85 \\
& Stot & 70 & 137 & 86 \\
& & & & \\
& Chao $_{1}$ & 58 & 71 & 86 \\
& Jackknife $_{1}$ & 79 & 97 & 110 \\
& Bootstrap $_{\text {Sesgo }}$ & 67 & 67 & 96 \\
& & & & \\
& Chao $_{1}$ & -0.409 & -0.622 & -0.219 \\
& Jackknife $_{1}$ & -0.193 & -0.487 & -0.008 \\
& Bootstrap $_{*}$ & -0.369 & -0.678 & -0.179 \\
& & & & \\
& Chao $_{1}$ & 0.203 & 0.399 & 0.079 \\
& Jackknife $_{1}$ & 0.125 & 0.267 & 0.073 \\
& Bootstrap & 0.222 & 0.482 & 0.147 \\
\hline
\end{tabular}
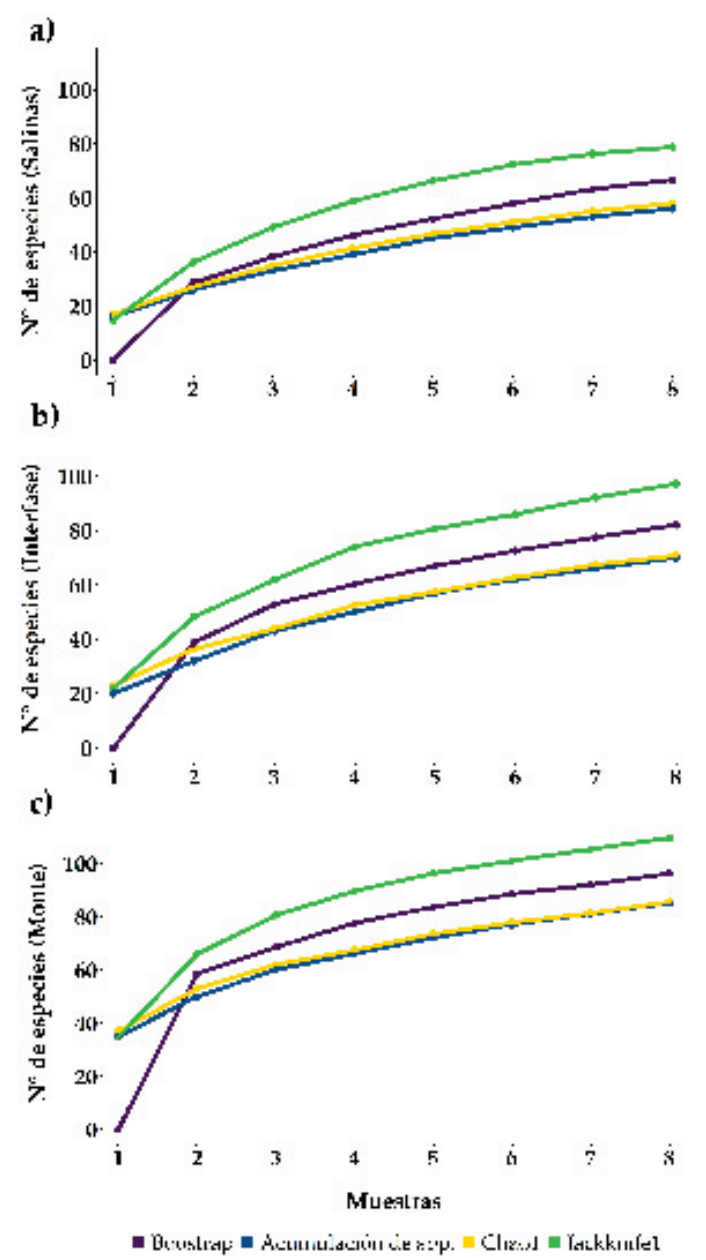

Figura 2. Curvas de acumulación de especies de la riqueza de los cuatro órdenes más diversos de Insecta (Hymenoptera, Coleoptera, Hemiptera, Diptera) (Sobs) y de los estimadores de riqueza de especies (Bootstrap, $\mathrm{Chao}_{1}$, Jackknife ${ }_{1}$ ) durante la temporada cálida (primavera y verano) para los tres ambientes: a) Salinas, b) Interfaz y c) Monte.

Figure 2. Species accumulation curves of the four most abundant orders of Insecta (Hymenoptera, Coleoptera, Hemiptera, Diptera) (Sobs) and the non-parametric species richness estimators used (Bootstrap, Chao Jackknife ${ }_{1}$ ) during the warm season (Spring and Summer) within the three environments: a) Salinas, b) Interface y c) Monte.

La abundancia de artrópodos difirió significativamente entre los ambientes ( $\mathrm{F}=15.759 ;$ g.l. $=2 ; P=0.001$ ), pero no entre las estaciones ( $\mathrm{F}=2.46$; g.l. $=1 ; P=0.152)$, aunque el verano registró una mayor cantidad de individuos (4802 individuos) respecto a primavera (3578). El Monte fue el ambiente con mayor abundancia de artrópodos (4317), diferenciándose de los otros ambientes (Interfaz: 2761 individuos y Salinas: 1302) 


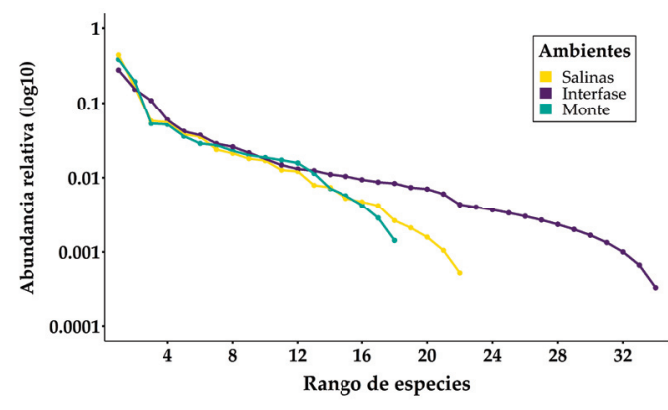

Figura 3. Curvas de rango-abundancia de especies de insectos epígeos registradas en la región de Los Llanos riojanos durante la temporada cálida (primavera y verano).

Figure 3. Rank-abundance curves of species for the epigaeic insects in Los Llanos region (La Rioja province) during the warm season (Spring and Summer).

(Figura 4a). Los individuos se agruparon en 10 taxa, de los cuales el más abundante fue Insecta (5902 individuos), representando $70.43 \%$ del total, seguido por Acari (1481, con el $17.67 \%)$, Collembola (473, con 5.64\%), Araneae $(419$, con el $5 \%)$ y Otros $(105$, con el $1.26 \%$ del total), que incluye Solifugae, Scorpionidae, Crustacea (Malacostraca), Isopoda, Pseudoscorpionida, Opiliones y Chilopoda. Dentro de los artrópodos, los insectos se agruparon en 14 órdenes, 31 superfamilias, 35 familias y 114 especies/morfoespecies, siendo Hymenoptera el orden más abundante (Anexo 1). El Monte fue el ambiente con la mayor abundancia total de insectos (3120 individuos), y se diferenció significativamente respecto a los otros ambientes $(\mathrm{F}=13.230$; g.1.=2; $P=0.002)$. La Interfaz fue el segundo ambiente más abundante (1932 individuos), mientras que las Salinas registró el menor número de individuos (850) (Figura 4b). La abundancia total de insectos también difirió significativamente entre las estaciones ( $\mathrm{F}=11.712 ;$ g.l. $=1 ; P=0.008)$, siendo mayor el número de insectos registrados en verano (3.811) respecto a primavera (2091). Por otro lado, la abundancia por familia de insectos difirió significativamente entre los ambientes y estaciones, habiendo un efecto significativo de la interacción entre estos dos factores $(\mathrm{F}=3.750$; g.l. $=74 ; P<0.001)$, destacándose la abundancia de Formicidae en el Monte durante el verano. Se registró un mayor número de individuos de las familias de insectos durante el muestreo de verano (3288 individuos; $65.08 \%$ del total), respecto a primavera (1709 individuos; $34.20 \%$ del total). Formicidae fue la familia de mayor abundancia (4997 individuos; $89.26 \%$ del total de insectos) y riqueza de especies (37 especies / a)

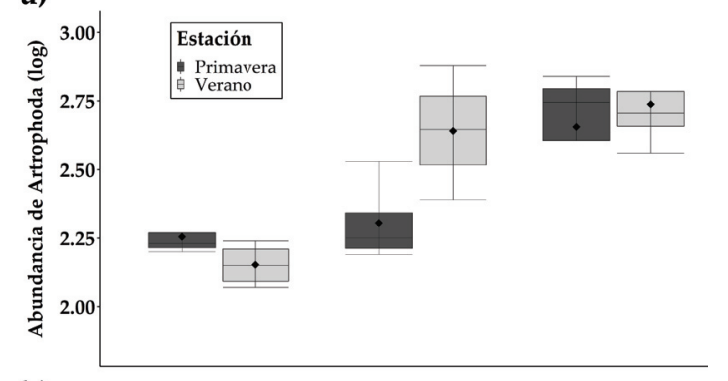

b)

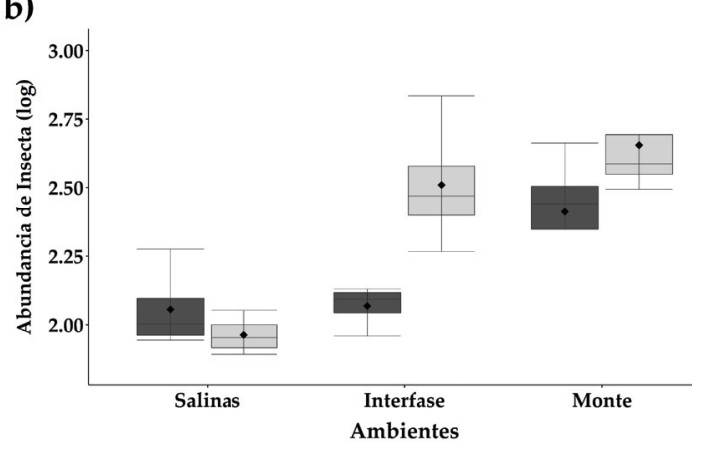

Figura 4. Abundancia de artrópodos (a) e insectos (b), en los tres ambientes (Salinas, Interfaz y Monte), en la región de Los Llanos (La Rioja), durante la temporada cálida (primavera y verano). Se indican la mediana (línea horizontal negra) y la media (rombo) para cada ambiente y estación. El 50\% de los valores centrales se encuentran dentro de cada caja, mientras que las líneas verticales representan los cuartiles superior e inferior.

Figure 4. Abundance of arthropods (a) and insects (b) in the three sampling environments (Salinas, Interface and Monte) in Los Llanos region (La Rioja province), during warm season (Spring and Summer). The median (horizontal black line) and the average (rhomb) are indicated for each environment and sampling period. The $50 \%$ of the central values are inside each box, while the vertical lines represent the upper and lower quartiles.

Tabla 2. Categorías de dominancia considerando el total de individuos (abundancia) de cada taxa. Referencias: Eu=eudominante $(\mathrm{CD}>10 \%), \mathrm{D}=$ dominante $(5 \%<\mathrm{CD} \leq 10 \%), \mathrm{SD}=$ subdominante $(2 \%<\mathrm{CD} \leq 5 \%)$, $\mathrm{E}=$ eventual $(1 \%<\mathrm{CD} \leq 2 \%), \mathrm{R}=$ rara $(\mathrm{CD} \leq 1 \%)$.

Table 2. Dominancy categories considering total abundance of individuals of each taxa. Referencies: $\mathrm{Eu}=$ eudominant $(\mathrm{CD}>10 \%), \mathrm{D}=\operatorname{dominant}(5 \%<\mathrm{CD} \leq 10 \%)$, $\mathrm{SD}=$ subdominant $\quad(2 \%<\mathrm{CD} \leq 5 \%), \quad \mathrm{E}=$ eventual $(1 \%<\mathrm{CD} \leq 2 \%), \mathrm{R}=$ rare $(\mathrm{CD} \leq 1 \%)$.

\begin{tabular}{lcccc}
\hline $\mathrm{CD}$ & Orden & Familia & $\begin{array}{c}\text { Especies/ } \\
\text { morfoespecies }\end{array}$ & Subfamilia \\
\hline $\mathrm{Eu}$ & $7 \%$ & $3 \%$ & $3 \%$ & $50 \%$ \\
$\mathrm{D}$ & - & - & $2 \%$ & - \\
$\mathrm{SD}$ & $14 \%$ & - & - & $17 \%$ \\
$\mathrm{E}$ & $22 \%$ & $6 \%$ & $7 \%$ & - \\
$\mathrm{R}$ & $57 \%$ & $91 \%$ & $88 \%$ & $33 \%$ \\
\hline
\end{tabular}


Tabla 3. Índices de diversidad de Shannon $\left(\mathrm{H}^{\prime}\right)$, de dominancia de Simpson (DS) y de equidad de Shannon (H'eq) para los tres ambientes (Salinas, Interfaz, Monte) en la región de Los Llanos (La Rioja), durante la temporada cálida (primavera y verano).

Table 3. Shannon diversity index $\left(\mathrm{H}^{\prime}\right)$, Simpson dominance index (DS) and Shannon evenness index (H'eq) for the three environments (Salinas, Interface, Monte) in Los Llanos region (La Rioja province) during the warm season (Spring and Summer).

\begin{tabular}{lcccc}
\hline Estación & Ambiente & $\begin{array}{c}\text { Diversidad } \\
\left(\mathrm{H}^{\prime}\right)\end{array}$ & $\begin{array}{c}\text { Dominancia } \\
(\mathrm{DS})\end{array}$ & $\begin{array}{c}\text { Equidad } \\
\left(\mathrm{H}^{\prime} \mathrm{eq}\right)\end{array}$ \\
\hline \multirow{3}{*}{ Primavera } & Monte & 1.276 & 0.898 & 0.306 \\
& Interfaz & 0.787 & 0.733 & 0.477 \\
& Salinas & 0.952 & 0.803 & 0.393 \\
\multirow{2}{*}{ Verano } & Monte & 1.133 & 0.844 & 0.36 \\
& Interfaz & 0.973 & 0.764 & 0.455 \\
\hline
\end{tabular}

morfoespecies) dentro de los insectos, seguida por Tenebrionidae (105 individuos, $1.88 \%$ del total de insectos, 9 especies).

De los 14 órdenes de insectos, $57 \%$ se consideraron como raros; sólo Hymenoptera fue eudominante, Coleoptera y Hemiptera fueron subdominantes y Diptera, Thysanoptera y Thysanura fueron eventuales. A nivel de familia, Formicidae fue la única eudominante; mientras tanto, Tenebrionidae y Coccoidea fueron eventuales. Las subfamilias de Formicidae Dolichoderinae, Formicinae y Myrmicinae fueron eudominantes, Ectatomminae se consideró subdominante, mientras que Ecitoninae y Pseudomyrmecinae fueron consideradas raras. Finalmente, a un nivel más específico, 3 especies fueron eudominantes y 2 fueron dominantes (todas pertenecientes a Formicidae) (Tabla 2).

La composición de familias de insectos no difirió significativamente entre los ambientes, de acuerdo con el análisis de agrupamiento (cluster) $(\mathrm{F}=2.6932, P=0.26676)$, aunque hay una tendencia a que el Monte se separe de los

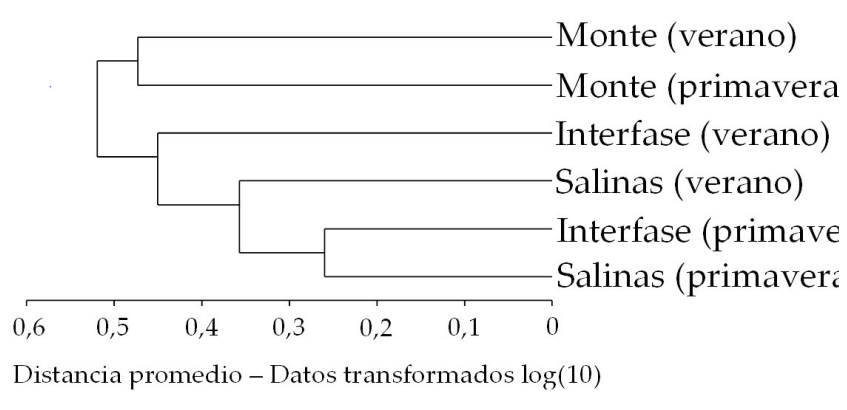

otros ambientes al considerar las familias más abundantes deinsectos(Figura5). Porotrolado, las familias recolectadas en el Monte fueron significativamente diferentes de aquellas que fueron exclusivas de las Salinas e Interfaz (eje 1 en el análisis de correspondencias [AC]), mientras que las familias más abundantes en primavera fueron significativamente diferentes a las familias más abundantes en verano (eje 2 del AC; $\chi^{2}=890.8124, P<0.000$ ). Los dos primeros ejes explicaron el $58.46 \%$ de la varianza total. El primer eje explicó el $32.16 \%$ de la varianza (eigenvalor $=0.267$ ), lo que sugiere un gradiente espacial, mientras que el segundo eje explicó el $26.39 \%$ (eigenvalor $=0.219$ ), sugiriendo un gradiente temporal. Dentro de las familias particulares de cada ambiente y estación se destacan Anthoporidae, Buprestidae, Corixidae y Syrphidae en el ambiente Salinas durante primavera y Dolichopodidae y Miridae en el mismo ambiente duranteel verano. Pompilidae y Sphecidae fueron específicas de Interfaz en primavera, mientras que Cecidomidae, Orthezíidae y Aphididae lo fueron durante el verano. Finalmente, las familias Alleculidae,

Figura 5. Análisis de cluster. Se indica el agrupamiento de tres ambientes (Salinas, Interfaz, Monte) en la región de Los Llanos (La Rioja), durante la temporada cálida (primavera y verano), según la abundancia por familias de insectos, considerando la distancia euclidiana promedio.

Figure 5. Cluster analysis showing the grouping for the three environments (Salinas, Interfaz, Monte) in Los Llanos region (La Rioja province) during the warm season (Spring and Summer), according to the abundance by insect families, considering average Euclidean distance. References: (primavera)=Spring, (verano) $=$ Summer. 


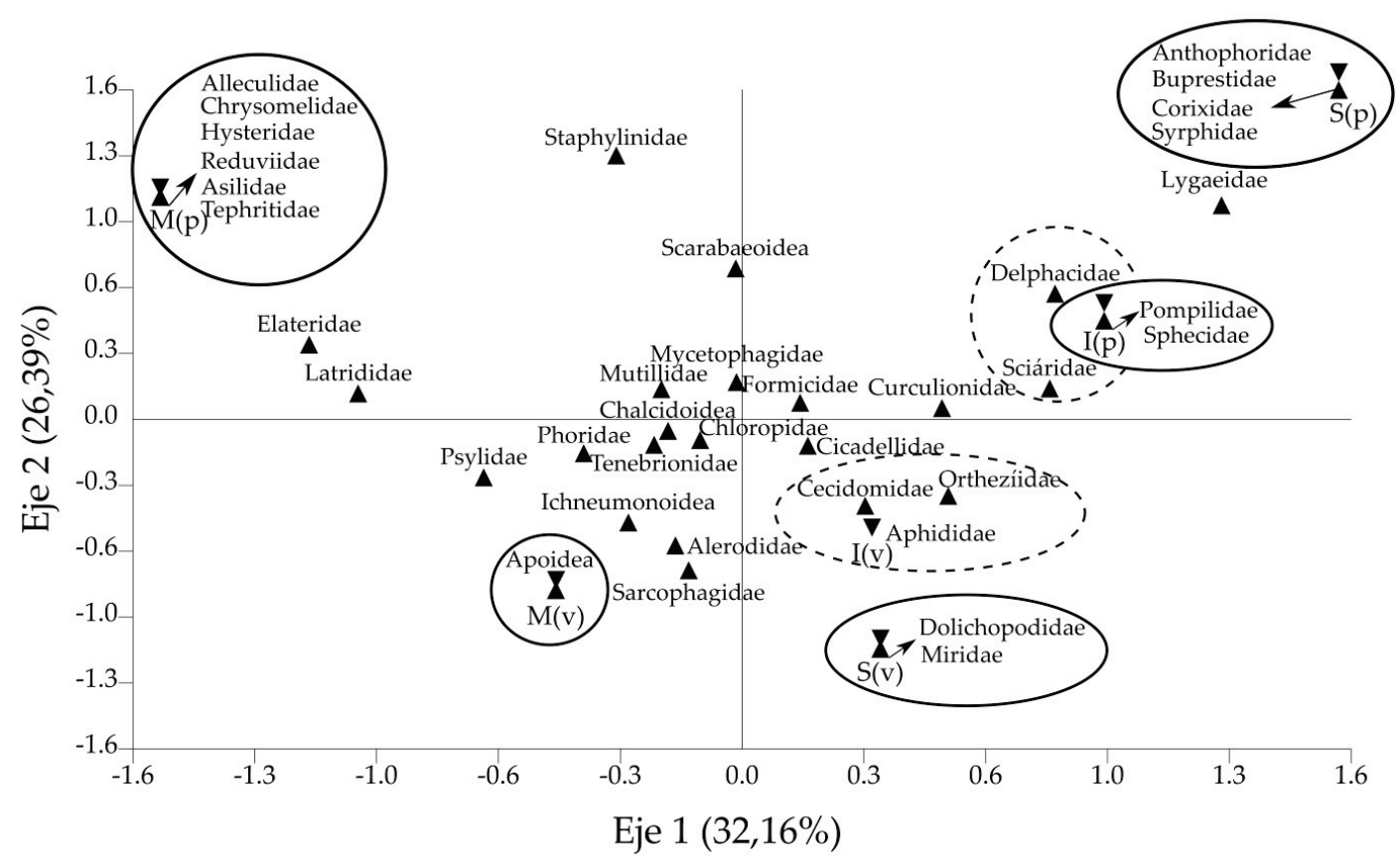

Figura 6. Análisis de correspondencia según la abundancia de las familias (y las superfamilias Ichneumonoidea, Scarabaeoidea, Chalcidoidea y Apoidea) registradas durante la temporada cálida (primavera y verano). Los triángulos indican las familias registradas, mientras que los triángulos invertidos indican el ambiente y la estación de muestreo. Las flechas indican aquellas familias encontradas en un ambiente y estación particular (triángulo). Los círculos continuos indican asociación directa al ambiente y estación particular, mientras que los círculos interrumpidos indican asociación con el ambiente. Referencias: $\mathrm{S}=$ Salinas, I=Interfaz, M=Monte, $(\mathrm{p})=$ primavera, $(\mathrm{v})=$ verano.

Figure 6. Correspondence analysis considering the abundance of families (and Icheneumonoidea, Scarabaeoidea, Chalcidoidea and Apoidea superfamilies) during the warm season (Spring and Summer). The triangles show the registered families, while the inverted triangles indicate the environment and sampling period. The arrows indicate those families collected in a particular sampling site and season (triangle). Continuous circles indicate direct association to the environment and particular sampling period, while interrupted circles indicate association with the environment. References: $\mathrm{S}=$ Salinas, I=Interphase, $\mathrm{M}=$ Monte, $(\mathrm{p})=$ Spring, $(\mathrm{v})=$ Summer.

Chrysomelidae, Hysteridae, Reduviidae, Asilidae y Tephritidae fueron exclusivas del ambiente Monte en primavera, y sólo Apoidea fue exclusiva de Monte en el verano. Otras familias como Elateridae, Latrididae y Psylidae se encontraron asociadas al Monte, pero no fueron exclusivas de ninguna estación climática. Cabe destacar que varias familias tales como Formicidae, Tenebrionidae, Cicadellidae, Mutilidae y Mycetophagidae (por mencionar algunas) no se asociaron a ningún ambiente en particular (Anexo $1 \mathrm{y}$ Figura 6).

Por otro lado, no se encontraron diferencias significativas en los valores de diversidad $\left(\mathrm{H}^{\prime}\right)$ ni equidad $\left(\mathrm{H}^{\prime} \mathrm{eq}\right)$ de insectos $(\mathrm{H}=15.18$; $P=0.096)$ entre ambientes ni estaciones $(\mathrm{H}=8.48$; $P=0.132$ ), aunque el Monte fue el ambiente con mayor diversidad y menor equidad (Tabla 3 ). La dominancia (DS) tampoco difirió entre ambientes ni estaciones, aunque el Monte tuvo una tendencia a presentar una mayor dominancia de insectos respecto a los otros ambientes $(\mathrm{H}=11 ; P=0.051)$. La Interfaz fue el ambiente con menor diversidad $\left(\mathrm{H}^{\prime}\right)$ y dominancia (DS), aunque presentó la mayor equidad, mientras que Salinas mostró valores intermedios entre los otros ambientes para los índices utilizados.

\section{DISCUSIÓN}

La variación en la diversidad (abundancia y riqueza de especies) y la composición del ensamble de artrópodos e insectos epígeos estuvo asociada al gradiente de vegetación Monte-Interfaz-Salinas en Los Llanos riojanos. El ambiente de mayor complejidad estructural en la vegetación (Monte) fue el de mayor abundancia de artrópodos y el de mayor abundancia y riqueza de insectos en ambas estaciones climáticas, mientras que los otros ambientes no presentaron grandes diferencias entre sí. Las diferencias encontradas en los 
patrones de diversidad y composición de artrópodos e insectos dentro de ellos, podrían deberse a características particulares de cada ambiente y a la composición florística que presentan las especies vegetales en estos ambientes según las estaciones climáticas. La estructuración del ensamble de artrópodos e insectos en la zona de Los Llanos parece responder de manera similar a patrones encontrados en otras zonas áridas, ya que la comunidad estuvo dominada por colémbolos, ácaros e insectos (particularmente hormigas), presentando estos últimos una predominancia de especies de abundancia intermedia.

La eficiencia de los estimadores no paramétricos evaluados en el presente estudio indican que $\mathrm{Chao}_{1}$ y Bootstrap serían buenos estimadores de riqueza de especies de insectos en nuestra área de estudio (Brose 2002; Walther and Moore 2005). Si bien se utilizaron más de cinco muestras para realizar estimaciones exactas y precisas (Brose 2002), relevar el total de especies de grupos muy diversos es prácticamente imposible (Chao et al. 2009). De hecho, los estimadores suelen no estabilizarse o alcanzar la asíntota incluso con valores muestrales altos (Novotný and Basset 2000), tal como ocurrió en nuestro estudio, probablemente debido a que cerca de la asíntota las especies añadidas suelen ser raras (Soberon and Llorente 1993). Aun así, los estimadores tendieron a estabilizarse, por lo que consideramos que el esfuerzo de muestreo se aproxima al necesario para obtener un registro completo de especies. Las curvas de acumulación de especies indican que luego de 8 muestreos, más de $50 \%$ del total de especies estimadas se habrán observado una vez, pero es probable encontrar más especies aumentando el esfuerzo de muestreo al extender el área o los períodos de muestreo (Hodge et al. 2017). Considerando la escasez de conocimiento en los patrones de diversidad y composición del ensamble de artrópodos e insectos en Los Llanos Riojanos, analizar los estimadores de riqueza nos brinda la posibilidad de proponer un buen estimador para esta zona árida y, además, estimar la riqueza total y generar muestreos completos. Estas ventajas optimizarían futuros estudios en la zona que son de vital importancia para establecer planes de manejo y conservación.

Por otro lado, la comunidad de insectos se caracterizó por una gran cantidad de especies de abundancia intermedia y por pocas especies muy abundantes y raras, ya que el modelo de mejor ajuste fue el log-normal truncado (Magurran 2004; McGill et al. 2007; Ulrich et al. 2016). Varios ensambles siguen este modelo en sus patrones de abundancia (Longino et al. 2002; Ulrich et al. 2010), que caracteriza comunidades con alta productividad y equidad, con poco recambio espacial y temporal de especies, y en asociación a ambientes heterogéneos (Magurran and Henderson 2003; Ulrich et al. 2016). Sin embargo, más de un modelo puede explicar la estructura de una comunidad (Magurran 2004; McGill et al. 2007), como ocurre en nuestro estudio, en el que el ambiente Monte ajusto a los modelos log normal truncado y log series para explicar la distribución de la abundancia de insectos. El modelo log series suele indicar comunidades con un alto grado de dispersión y recambio de especies o comunidades con muchas especies que han tenido un muestreo incompleto (Ulrich et al. 2016). Es probable que un mayor esfuerzo de muestreo permita obtener un registro más completo de la comunidad.

La diversidad de artrópodos, y de insectos en particular, estuvo asociada a características particulares del tipo de ambiente en un gradiente de vegetación. El ambiente Monte presentóla mayor riqueza y abundancia, lo cual puede atribuirse a la presencia de estructuras vegetales y especies leñosas como algarrobos, quebrachos y chañares, ausentes en los otros ambientes (Morello et al. 1973; Karlin et al. 2013). Estas estructuras clave y comunidades vegetales diversas crean microhábitats particulares (Fuster 2012; Rossi and Villagra 2003), aumentan la complejidad del sistema y generan mayores posibilidades de refugio y alimento, explicando la alta diversidad de artrópodos e insectos registrada en el Monte (Flores et al. 2004; Lagos 2004; Martínez 2018). Por otro lado, el ambiente Interfaz presentó abundancias intermedias entre los otros ambientes; en primavera la abundancia tanto de artrópodos como de insectos fue similar al ambiente Salinas, mientras que en verano fueron similares al ambiente Monte. Esta variabilidad en los patrones de abundancia encontrados en la Interfaz podría explicarse por diversas causas. Una de las explicaciones es su naturaleza como ambiente de transición, que presenta características conjuntas de los ambientes aledaños (Smith et al. 1997; Araújo 2002). Otra de las causas podría ser las variaciones estacionales que actúan de manera directa sobre la abundancia de insectos, modificando la cantidad de 
individuos en distintos ambientes (Flores 2004; Visintin 2012), o de manera indirecta, modificando la composición florística de los ambientes en primavera y verano tal como ocurre en otras zonas áridas (Peñaloza et al. 2012). Finalmente, las Salinas, aunque presentaron valores bajos de abundancia y riqueza, fueron similares a los registrados en Interfaz en primavera. Las áreas inundables o humedales de zonas áridas, tales como el ambiente Salinas en nuestro estudio, suelen presentar una alta biodiversidad (Neiff 2001; Ginzburg et al. 2005) y especies particulares adaptadas a las características propias del ambiente, como las halófitas (e.g., Allenrolfea patagónica [jume]). Estas especies aumentan la disponibilidad de recursos a las comunidades de insectos, ya que actúan como barrera contra el viento y el agua, favoreciendo la formación de suelo y permitiendo la instalación de otras especies menos halófitas (Ruiz Posse et al. 2007). De hecho, en las Salinas encontramos organismos propios de ambientes acuáticos tales como Corixidae y Odonata (Tripplehorn and Johnson 2005), siendo estos últimos muy utilizados como bioindicadores en estudios de manejo y monitoreo (Gerlach et al. 2013). En este sentido, este estudio brinda nueva evidencia que enfatiza la importancia de conocer y mantener la identidad de cada ambiente en relación a la entomofauna en zonas áridas/semiáridas.

Si se considera la composición taxonómica del ensamble de artrópodos, el grupo predominante dentro de ellos fueron los insectos, seguidos de colémbolos, ácaros y arañas, en concordancia con otros estudios realizados en zonas áridas (Flores et al. 2004; Peñaloza et al. 2012; González Reyes et al. 2012; Martínez 2018). La diversidad de artrópodos se puede explicar por la heterogeneidad vegetal, pero también por características propias de cada grupo; por ejemplo, la gran abundancia de colémbolos y ácaros en las trampas de caída puede deberse a su función como descomponedores de hojarasca (Gergócs and Hufnagel 2016). Por otro lado, la alta representatividad de insectos se debe a que son el grupo de mayor diversidad entre los invertebrados del suelo (Decaëns et al. 2006); puntualmente, Tenebrionidae (Coleóptera) y Formicidae (Hymenoptera) son los grupos de insectos más abundantes en diversas zonas áridas de la Argentina (Lagos 2004; Cheli et al. 2010; Peñaloza et al. 2012; Lescano et al. 2017). La abundancia de Tenebrionidae podría atribuirse a sus adaptaciones fisiológicas y comportamentales para vivir en ecosistemas áridos como, por ejemplo, mecanismos para evitar la pérdida de agua mediante criptonefridios y para aprovechar las precipitaciones durante la temporada de lluvias (Cloudsley-Thompson 2001; Flores et al. 2004). La gran abundancia y riqueza de Formicidae podría explicarse por su distribución cosmopolita, su gran diversidad y los polimorfismos en sus poblaciones, permitiendo encontrarlas en una gran variedad de ambientes y hábitats (Hölldobler and Wilson 1990). En los tres ambientes se encontraron individuos pertenecientes a las superfamilias Chalcidoidea, conocidos por sus hábitos parasitoides, y Scarabaeoidea, importantes descomponedores de materia orgánica entre otras funciones ecosistémicas (Tripplehorn and Johnson 2005), los cuales suelen ser utilizados para evaluar cambios en el hábitat (Gerlach et al. 2013). Otras familias de insectos registradas fueron Mutilidae, Elateridae y Curculionidae, las cuales suelen ser representativas en otras zonas áridas de la Argentina (Roig-Juñent et al. 2001; Flores 2004). Sin embargo, dado que Mutilidae y Curculionidae se encontraron en los tres ambientes, no poseerían potencial para ser especies indicadoras de los ambientes analizados. Aun así, diversos grupos encontrados en esteestudio(e.g., Acari, Aranae, Hymenoptera [Formicidae], Coleoptera [Scarabaeidae] y Díptera) son muy utilizados como indicadores del estado de conservación o de cambios en el ecosistema (Gerlach et al. 2013). Considerando el escaso conocimiento del ensamble de artrópodos en la zona de los Llanos y la situación actual del Chaco árido al cual pertenecen, los taxa registrados en este estudio poseen un gran potencial como grupos bioindicadores, brindando una herramienta de gran utilidad para diseñar planes de manejo o conservación.

Los artrópodos epígeos son esenciales en el ecosistema (Decaëns et al. 2006), ya sea como fuente de alimento para otras especies (Bernard et al. 1997) o como modificadores de aspectos funcionales de la comunidad (Whitford and Parker 1989) y de estructuras tróficas en ambientes áridos (Zeng et al. 2014). Dado que Los Llanos riojanos pertenecen al Chaco árido, una de las regiones más amenazadas actualmente por la pérdida de hábitat (Natenzon 1994; Grau et al. 2011), este estudio brinda herramientas para considerar a dicha región y a los grupos indicadores de artrópodos e insectos registrados, de gran 
interés para ser incluidos en planes de manejo y conservación.

AgradeCIMIENTOS. Este trabajo fue financiado con CICyT-UNLaR (Resol. CICyT Nº 043/
2014). Gracias a P. Lorenzo, B. Ordoñez, J. y L. Baudino, J. Izquierdo, N. Rivetti y N. Rocamundi por su ayuda en los muestreos, a J. Guido por el mapa del área de estudio y a L. Motta por sus valiosos comentarios.

\section{REFERENCIAS}

Acosta, L. E. 1995. The scorpions of the Argentinian Western Chaco. II. Community survey in the Llanos District. Biogeographica 7:187-196.

Álvarez, J. A., P. E. Villagra, B. E. Rossi, and E. M. Cesca. 2009. Spatial and temporal litterfall heterogeneity generated by woody species in the Central Monte desert. Plant Ecology 205:295-303. https://doi.org/10.1007/s11258-0099618-z.

Araújo, M. B. 2002. Biodiversity hotspots and zones of ecological transition. Conservation Biology 16:1662-1663. https: //doi.org/10.1046/j.1523-1739.2002.02068.x.

Araujo, P. I., L. Yahdjian, and A. T. Austin. 2012. Do soil organisms affect aboveground litter decomposition in the semiarid Patagonian steppe, Argentina? Oecologia 168:221-230. https://doi.org/10.1007/s00442-011-2063-4.

Ayal, Y. 2007. Trophic structure and the role of predation in shaping hot desert communities. Journal of Arid Environments 68:171-187. https://doi.org/10.1016/j.jaridenv.2006.05.013.

Bardgett, R. D., and D. A. Wardle. 2010. Aboveground- Belowground Linkages. Biotic Interactions, Ecosystem Processes, and Global Change. Oxford University Press.

Basualdo, C. V. 2011. Choosing the best non-parametric richness estimator for benthic macroinvertebrates databases. Revista de la Sociedad Entomológica Argentina 70(1-2):27-38.

Bernard, J. B., M. E. Allen, and D. E. Ullrey. 1997. Feeding captive insectivorous animals: nutritional aspects of insects as food. Nutrition Advisory Group Handbook. Pp. 1-7.

Biani, N. B., J. L. Vesprini, and D. E. Prado. 2004. Conocimiento sobre el Gran Chaco Argentino en el Siglo XX. Pp. 1-24 en Ecología y Manejo de los Bosques de Argentina. Cap. 7.

Biurrun, F. N., W. D. Agüero, and D. F. Teruel. 2012. Consideraciones fitogeográficas sobre la vegetación de Los Llanos de La Rioja. Serie: Estudios sobre el Ambiente y el Territorio. INTA. ISSN: 1853-3647.

Brose, U. 2002. Estimating species richness of pitfall catches by non-parametric estimators. Pedobiologia 46:101-107. https://doi.org/10.1078/0031-4056-00117.

Cardoso, J. D. C., M. B. de Paula, A. Fernandes, E. dos Santos, M. A. B. de Almeida, D. F. da Fonseca, and M. A. M. Sallum. 2011. Ecological aspects of mosquitoes (Diptera: Culicidae) in an Atlantic forest area on the north coast of Rio Grande do Sul State, Brazil. Journal of Vector Ecology 36:175-186. https://doi.org/10.1111/j.1948-7134.2011.00155.x.

Chao, A., R. K. Colwell, C. W. Lin, and N. J. Gotelli. 2009. Sufficient sampling for asymptotic minimum species richness estimators. Ecology 90:1125-1133. https://doi.org/10.1890/07-2147.1.

Cheli, G. H., J. C. Corley, O. Bruzzone, M. del Brío, F. Martínez, N. M. Román, and I. Ríos. 2010. The Ground-Dwelling Arthropod Community of Península Valdés in Patagonia, Argentina. Journal of Insect Science 10:1-16. https:// doi.org/10.1673/031.010.5001.

Cloudsley-Thompson, J. 2001. Thermal and water relations of desert beetles. Naturwissenschaften 88:447-460. https: / / doi.org/10.1007/s001140100256.

Coirini, R., and U. Karlin. 2000. Potencialidad económica de bosques con algarrobos I: Propuesta de uso múltiple para el Chaco Árido. Multequina 9:133-145.

Coirini, R. O., M. S. Karlin, and G. J. Reati. 2010. Manejo sustentable del ecosistema salinas grandes, chaco árido. R. O. Coirini, M. S. Karlin and G. J. Reati (eds.). Encuentro Grupo Editor. Pp. 322.

Decaëns, T., J. J. Jiménez, C. Gioia, G. J. Measey, and P. Lavelle. 2006. The values of soil animals for conservation biology. European Journal of Soil Biology 42:S23-S38. https://doi.org/10.1016/j.ejsobi.2006.07.001.

Di Rienzo, J. A., F. Casanoves, M. G. Balzarini, L. González, M. Tablada, and C. W. Robledo. 2009. InfoStat: statistical software.

Fernández, F., and M. J. Sharkey. 2006. Introducción a los Hymenoptera de la Región Neotropical. Sociedad Colombiana de Entomología y Universidad Nacional de Colombia, Bogotá.

Fisher, B. L., and S. P. Cover. 2007. Ants of North America: a guide to the genera. Univ. of California Press.

Flores, G. E., S. J. Lagos, and R. -J. Sergio. 2004. Artrópodos epígeos que viven bajo la copa de algarrobo (Prosopis flexuosa) en la reserva Telteca (Mendoza, Argentina). Multequina 13:71-90.

Fuster, A. 2012. Especies de hormigas asociadas a Prosopis ruscifolia Griseb. en ambientes salinos del Chaco Semiárido. Quebracho 20:29-38.

Gardner, S. M., M. R. Cabido, G. R. Valladares, and S. Díaz. 1995. The influence of habitat structure on arthropod diversity in Argentine semi-arid Chaco forest. Journal of Vegetation Science 6:349-356. https://doi.org/10.2307/3236234.

Gergócs, V., and L. Hufnagel. 2016. The effect of microarthropods on litter decomposition depends on litter quality. European Journal of Soil Biology 75:24-30. https://doi.org/10.1016/j.ejsobi.2016.04.008.

Gerlach, J., M. Samways, and J. Pryke. 2013. Terrestrial invertebrates as bioindicators: an overview of available taxonomic groups. Journal of Insect Conservation 17:831-850. https:// doi.org/10.1007/s10841-013-9565-9. 
Ginzburg, R., J. Adamoli, P. Herrera, and S. Torrella. 2005. Los Humedales del Chaco: Clasificación, Inventario y Mapeo a Escala Regional. Miscelánea 14:121-138.

González Reyez, A. X, J. A. Corronca, and N. M. Arroyo. 2012. Differences in alpha and beta diversities of epigeous arthropod assemblages in two ecoregions of northwestern Argentina. Zoological Studies 51:1367-1379.

Gotelli, N., and R. Colwell. 2011. Estimating species richness. Pp. 39-54 in Biological Diversity. Frontiers in Measurement and Assessment. Oxford University Press, United Kingdom. https://doi.org/10.1046/j.1461-0248.2001.00230.x.

Gotelli, N. J., and R. K. Colwell. 2001. Quantifying Biodiversity: Procedures and Pitfalls in the Measurement and Comparison of Species Richness. Ecology Letters 4:379-391.

Grau, R., I. Gasparri, and M. Gasparri. 2011. Cambio y eficiencia de uso del territorio en el Chaco argentino: el conflicto entre producción de alimentos y conservación de la naturaleza en distintas escalas. Valoración de Servicios Ecosistémicos: Conceptos, herramientas y aplicaciones para el ordenamiento territorial. MAGyP.

Henderson, P. A., and R. M. H. Seaby. 1997. Species diversity and richness. Version 1.2 PISCES Conservation Limited. Lymington, United Kingdom.

Hodge, S., N. Curtis, C. J. Vink, J. Marris, and S. D. J. Brown. 2017. Native arthropods on exotic sand dune flowers: consideration of sample size and number for investigating rare species and sparse communities. Arthropod-Plant Interactions 11:691-701. https:/ / doi.org/10.1007/s11829-017-9521-9.

Hölldobler, B., and E. O. Wilson. 1990. The ants. Harvard University Press. https:/ /doi.org/10.1007/978-3-662-103067.

Hortal, J., P. A. V. Borges, and C. Gaspar. 2006. Evaluating the performance of species richness estimators: Sensitivity to sample grain size. Journal of Animal Ecology 75:274-287. https://doi.org/10.1111/j.1365-2656.2006.01048.x.

Karlin, M. S., U. O. Karlin, R. O. Coirini, G. J. Reati, and R. M. Zapata. 2013. El Chaco árido. Universidad Nacional de Córdoba. https:/ / doi.org/10.1155/2013/945190.

Kovach, W. L. 1999. MVSP - A multivariate statistical Package for Windows, ver. 3.1. Kovach Computing Services, Pentraeth, Wales, UK. Pp. 137.

Lagos, S. J. 2004. Diversidad biológica de las comunidades epígeas de artrópodos en áreas pastoreadas y no pastoreadas del Monte (Argentina). Tesis Doctoral. Universidad Nacional de Cuyo, Mendoza.

Lescano, M. N., L. Elizalde, V. Werenkraut, G. I. Pirk, and G. E. Flores. 2017. Ant and tenebrionid beetle assemblages in arid lands: Their associations with vegetation types in the Patagonian steppe. Journal of Arid Environments 138: 51-57. https://doi.org/10.1016/j.jaridenv.2016.12.002.

Longino, J. T., J. Coddington, and R. K. Colwell. 2002. the Ant Fauna of a Tropical Rain Forest: Estimating Species. Ecology 83:689-702. https://doi.org/10.1890/0012-9658(2002)083[0689:TAFOAT]2.0.CO;2.

Magurran, A. 2004. Measuring Biological Diversity. Blackwell Science Ltd.

Magurran, A. E., and P. A. Henderson. 2003. Explaining the excess of rare species in natural species abundance distributions. Nature 422:714-716. https://doi.org/10.1038/nature01547.

Martín-Piera, F., and J. M. Lobo. 2000. Diagnóstico Sobre El Conocimiento Sistemático Y Biogeográfico De Tres Órdenes De Insectos Hiperdiversos En España: Coleoptera, Hymenoptera Y Lepidoptera. Hacia un Proyecto CYTED para el Inventario y Estimación de la Diversidad Entomológica en Iberoamérica: PrIBES-2000. 1:287-308.

Martínez, F. J. 2018. Ensambles de artrópodos asociados a arbustos nativos del noreste de la Patagonian: su relación con la complejidad estructural de la vegetación y el pastoreo ovino. Facultad de Ciencias Naturales y Museo de la Universidad Nacional de La Plata (FCNyM-UNLP).

Mattoni, C. I., and L. E. Acosta. 1997. Scorpions of the insular Llanos district (Province of La Rioja, Argentina) and their zoogeographical links. Biogeographica 73:67-80.

Maurer, B. A., and B. J. McGill. 2011. Measurement of species diversity. Biological Diversity: Frontiers in measurement and assessment. Oxford University Press. Pp. 55-65.

McGill, B. J., R. S. Etienne, J. S. Gray, D. Alonso, M. J. Anderson, H. K. Benecha, M. Dornelas, B. J. Enquist, J. L. Green, F. He, A. H. Hurlbert, A. E. Magurran, P. A. Marquet, B. A. Maurer, A. Ostling, C. U. Soykan, K. I. Ugland, and E. P. White. 2007. Species abundance distributions: Moving beyond single prediction theories to integration within an ecological framework. Ecology Letters 10:995-1015. https:/ / doi.org/10.1111/j.1461-0248.2007.01094.x.

Morello, J. H., J. Protomastro, L. Sancholuz, and C. Blanco. 1973. Estudio macroecológico de los Llanos de La Rioja. APN, Administración de Parques Nacionales, Secretariat de Agricultura, Ganadería y Pesca, Ministerio de Economía.

Moreno, C. E. 2001. Métodos para medir la biodiversidad. MT-Manuales y Tesis SEA 1:84 pp.

Natenzon, C. E. 1994. La tala del bosque en Los Llanos de La Rioja (1900-1960). Desarrollo Económico 34:263-284. https://doi.org/10.2307/3467319.

Neiff, J. J. 2001. Humedales de la Argentina: sinopsis, problemas y perspectivas futuras. El agua en Iberoamérica. Funciones de los humedales, calidad de vida y agua segura. Pp. 83-112.

Novotný, V., and Y. Basset. 2000. Rare species in communities of tropical insect herbivores: Pondering the mystery of singletons. Oikos 89:564-572. https://doi.org/10.1034/j.1600-0706.2000.890316.x.

Oyarzabal, M., J. Clavijo, L. Oakley, F. Biganzoli, P. Tognetti, I. Barberis, H. M. Maturo, R. Aragón, P. I. Campanello, D. Prado, M. Oesterheld, and R. J. C. León. 2018. Unidades de vegetación de la Argentina. Ecología Austral 28:040-063. https://doi.org/10.25260/EA.18.28.1.0.399.

Peñaloza, O. A., J. Corronca, and M. Balzarini. 2012. Descripción de artrópodos epigeos en dos ambientes del Parque Nacional Talampaya, La Rioja, Argentina. UNLaR Ciencia 1:17-23. 
Ragonese, A. E., and J. C. Castiglioni. 1970. La vegetación del Parque Chaqueño. Bol Soc Arg Bot 11(supl.):133-160. Released, S. I. 2008. SPSS statistics for windows, version 17.0. Chicago: SPSS Inc.

Roig-Juñent, S., G. Flores, S. Claver, G. Debandi, and A. Marvaldi. 2001. Monte Desert (Argentina): Insect biodiversity and natural areas. Journal of Arid Environments 47:77-94. https://doi.org/10.1006/jare.2000.0688.

Roig, F. A., S. Roig-Juñent, and V. Corbalán. 2009. Biogeography of the Monte Desert. Journal of Arid Environments 73:164-172.

Rossi, B. E., and P. E. Villagra. 2003. Effects of Prosopis exuosa on soil properties and the spatial pattern of understorey species in arid Argentina. Journal of Vegetation Science 14:543-550. https://doi.org/10.1658/1100-9233(2003)014[0543: EOPFOS]2.0.CO;2. https://doi.org/10.1111/j.1654-1103.2003.tb02181.x.

Ruiz Posse, E., U. Karlin, and E. Buffa. 2007. Ambientes de las salinas grandes de Catamarca, Argentina. Multequina 16:123-137.

Smith, T. B., R. K. Wayne, D. J. Girman, and M. W. Bruford. 1997. A Role for Ecotones in Generating Rainforest Biodiversity. Science 276:1855-1857. https://doi.org/10.1126/science.276.5320.1855.

Soberon, J., and J. Llorente. 1993. The use of species accumulation functions for the prediction of species richness. Conservation biology: the journal of the Society for Conservation Biology 7:480-488. https://doi.org/10.1046/j.15231739.1993.07030480.x.

Stein, A., K. Gerstner, and H. Kreft. 2014. Environmental heterogeneity as a universal driver of species richness across taxa, biomes and spatial scales. Ecology Letters 17:866-880. https://doi.org/10.1111/ele.12277.

Tews, J., U. Brose, V. Grimm, K. Tielbörger, M. C. Wichmann, M. Schwager, and F. Jeltsch. 2004. Animal species diversity driven by habitat heterogeneity/diversity: The importance of keystone structures. Journal of Biogeography 31:79-92. https://doi.org/10.1046/j.0305-0270.2003.00994.x.

Tripplehorn, C. A., and N. F. Johnson. 2005. Borror and DeLong's introduction to the study of insects. Thomson Brooks/Cole, Belmont, California.

Ulrich, W., M. Ollik, and K. I. Ugland. 2010. A meta-analysis of species-abundance distributions. Oikos 119:11491155.

Ulrich, W., S. Soliveres, A. D. Thomas, A. J. Dougill, and F. T. Maestre. 2016. Environmental correlates of species rank - abundance distributions in global drylands. Perspectives in Plant Ecology, Evolution and Systematics 20:56-64. https://doi.org/10.1111/j.1600-0706.2009.18236.x.

Visintin, M. A. 2012. Estructura y dinámica de la comunidad de Culicidae (Díptera) en el Arco Sur de La Laguna de Mar Chiquita, Córdoba, Argentina. Tesis de Grado. Pp. 213.

Walther, B. A., and Moore, J. L. 2005. The concepts of bias, precision and accuracy, and their use in testing the performance of species richness estimators, with a literature review of estimator performance. Ecography 28(6):815-829. https: //doi.org/10.1111/j.2005.0906-7590.04112.x.

Whitford, W. G., and L. W. Parker. 1989. Contributions of soil fauna to decomposition and mineralization processes in semiarid and arid ecosystems. Arid Soil Research and Rehabilitation 3:199-215. https://doi.org/10.1080/ 15324988909381199.

Zeng, Z., J. Bi, S. Li, S. Chen, D. A. Pike, Y. Gao, and W. Du. 2014. Effects of habitat alteration on lizard community and food web structure in a desert steppe ecosystem. Biological Conservation 179:86-92. https://doi.org/10.1016/ j.biocon.2014.09.011.

Zuazo, V. H. D., and C. R. R. Pleguezuelo. 2009. Soil-Erosion and Runoff Prevention by Plant Covers: A Review. Pp. 785-811. Sustainable Agriculture. Springer, Dordrecht. https://doi.org/10.1007/978-90-481-2666-8_48. 